\title{
Right hydrothorax misconceived as atelectasis after left internal jugular vein catheterization -A case report-
}

\author{
Hong Sik Lee, Chu Hwan Seo, Jong Kwon Jung, Jeong Uk Han, Seong Jin Jeong, and Hyun \\ Kyoung Lim
}

Department of Anesthesiology and Pain Medicine, Inha University College of Medicine, Incheon, Korea

\begin{abstract}
Central vein catheterization is a common procedure for monitoring the central venous pressure, securing vascular access, administrating vasoactive drugs and removing air embolisms. However, many complications can occur, such as vessel injury, pneumothorax, hydrothorax, nerve injury, arrhythmia and infection at the insertion site. We encountered an unusual complication of a localized right hydrothorax that was initially misinterpreted as an atelectasis after left internal jugular vein catheterization and right lateral positioning for a left lower lobectomy. (Korean J Anesthesiol 2010; 58: 87 90)
\end{abstract}

\section{Key Words: Central venous catheterization, Complications, Hydrothorax.}

Central venous catheterization is a procedure performed in operating rooms, emergency rooms, and intensive care units to monitor the central venous pressure (CVP), provide secure rapid fluid resuscitation, injection ports of administrating drugs, and secure vascular access in cases of a difficulty in peripheral venous catheterization. In addition, also it is performed to insert pulmonary artery catheters, monitor the pulmonary arterial pressure, and remove air emboli. However, potentially complications occur in $15 \%$ of central venous catheterizations [1], such as vessel injury, pneumothorax, hydrothorax, nerve injury, arrhythmia, pulmonary embolism and infection at the insertion site or through the catheter $[1,2]$. The authors report a case of localized right hydrothorax that was initially misinterpreted as atelectasis after left internal jugular vein catheterization.

\section{Case Report}

A chest CT scan of a 63-year-old, 62 kg, $156 \mathrm{~cm}$ female patient revealed a lung tumor. She was admitted into hospital for a left lower lobectomy. Her medical history only showed well-managed hypertension and diabetes mellitus. The preoperative blood tests appeared normal. A simple chest $X$-ray revealed a left lower lobe solitary pulmonary nodule and a calcified nodule in the right lung.

After inducing general anesthesia, endotracheal intubation was performed with a double-lumen tube for one-lung ventilation. After confirming the modified Allen test, an arterial catheter was inserted at the right radial artery for continuous monitoring of the blood pressure and arterial blood gas

Received: June 26, 2009. Revised: 1st, August 11, 2009, 2nd, August 27, 2009. Accepted: September 6, 2009.

Corresponding author: Hyun Kyoung Lim, M.D., Department of Anesthesiology and Pain Medicine, Inha University College of Medicine, 7-206, Sinheungdong 3ga, Jung-gu, Incheon 400-711, Korea. Tel: 82-32-890-3968, Fax: 82-32-881-2476, E-mail: hkliman@inha.ac.kr

(c) This is an open-access article distributed under the terms of the Creative Commons Attribution Non-Commercial License (http:// creativecommons.org/licenses/by-nc/3.0/), which permits unrestricted non-commercial use, distribution, and reproduction in any medium, provided the original work is properly cited. 
analysis (ABGA). Subsequently, a central venous catheter (CVC) was inserted into the left internal jugular vein. When operating on the left lung, placing the patient in the right lateral recumbent position allows manipulation of the left internal jugular venous catheter. In addition, it is possible to quickly recognize any mechanical damage to the operation site when inserting the CVC.

To insert the CVC, the patient's head was lowered $5^{\circ}$ and the neck was rotated approximately $15^{\circ}$ to the right. The internal jugular vein was punctured with an 18-gauge needle with ultrasound guidance. After vein puncture, a J-tip guide wire was inserted and the needle was removed, the insertion site for the catheter was dilated with a venodilator, and a 7 Fr. central vein catheter (Two-lumen Central Venous Catheterization Set with Vantex ${ }^{\circledR}$, Edward Lifescience Co., USA) was inserted. Subsequently, the aspiration of blood by the two lumens of the catheter was observed, and the catheter was secured at a depth of $18 \mathrm{~cm}$ below the skin after checking the CVP waves.

The patient was placed in the right lateral recumbent position for surgery. Surgery was performed after performing one lung ventilation. The concentration of sevoflurane and the dose of the remifentanil infusion were adjusted accordingly to the vital signs, and the ventilator was set in pressure-control mode (peak air way pressure at $21 \mathrm{mmHg}$, respiratory rate at 11 , inspiratory to expiratory time ratio at $1: 2$ ). After starting one lung ventilation, the $\mathrm{ABGA}$ was $\mathrm{pH} 7.47, \mathrm{PaCO}_{2} 31 \mathrm{mmHg}$, $\mathrm{PaO}_{2} 264 \mathrm{mmHg}$, and bicarbonate (BE) $22.6 \mathrm{mmol} / \mathrm{L}$ at $\mathrm{FiO}_{2}$ 1.0. The respiration rate was adjusted so that $\mathrm{PaCO}_{2}$ would be between 36 and $40 \mathrm{mmHg}$. There were no abnormal findings during surgery, and the surgeon inserted the left chest tube. The total time under anesthesia care was 7 hours and 40 minutes. The CVP was kept at 6-11 $\mathrm{mmHg}$, and no changes in the wave forms were observed. Blood loss during surgery was estimated to be approximately $50 \mathrm{ml}$ with a urine output at $350 \mathrm{ml}$. The total amount of fluid administered was 1,500 $\mathrm{ml}$, of which $200 \mathrm{ml}$ was a saline and $400 \mathrm{ml}$ was pentastarch (Pentaspan ${ }^{\circledR}$, Jeil Pharm, S. Korea) injected into the central vein.

Immediately after surgery, a chest X-ray was taken with the patient in the supine position which led to an abnormal finding in the right lung (Fig. 1A). Atelectasis was found in the right upper lobe apex after obtaining the opinion of a thoracic surgeon. Accordingly, the right bronehus was checked with a fiberoptic bronchoscope. There was no secretion or blood clot, nor was there any blockage found in the airway found, so positive-pressure ventilation was performed. An additional chest X-ray showed normal findings (Fig. 1B). Consequently, the muscle relaxants were reversed, spontaneous respiration was restored, and the double-lumen tube was extubated. The patient was provided oxygen via a mask and transported to the intensive care unit (ICU) for observation.

On the day of surgery, the patient in the ICU reported no other pain or discomfort other than that at the operation site. Her vital signs were normal. She did not complain of breathing difficulty. On postoperative day 1 , her vital signs were stable. However, on a simple chest X-ray, the opacity of the right lung increased and the mediastinum was shifted to the left (Fig. 2). Consequently, a thoracentesis was performed. $700 \mathrm{ml}$ of pleural fluid was drained. The glucose level was $107 \mathrm{mg} / \mathrm{dl}$ with a protein level $<1 \mathrm{~g} / \mathrm{dl}$, so it was estimated to be effusion fluid. Two days after surgery in the morning, the breathing sounds from the right lower region reduced. A chest $X$-ray revealed an increase in opacity of the right lung. A 28 F. chest tube was inserted and 1,400 ml of pleural fluid was drained. The cause of the right-side pleural effusion was believed to be due to movement of the central vein catheter, so the catheter was removed. After observing a decrease
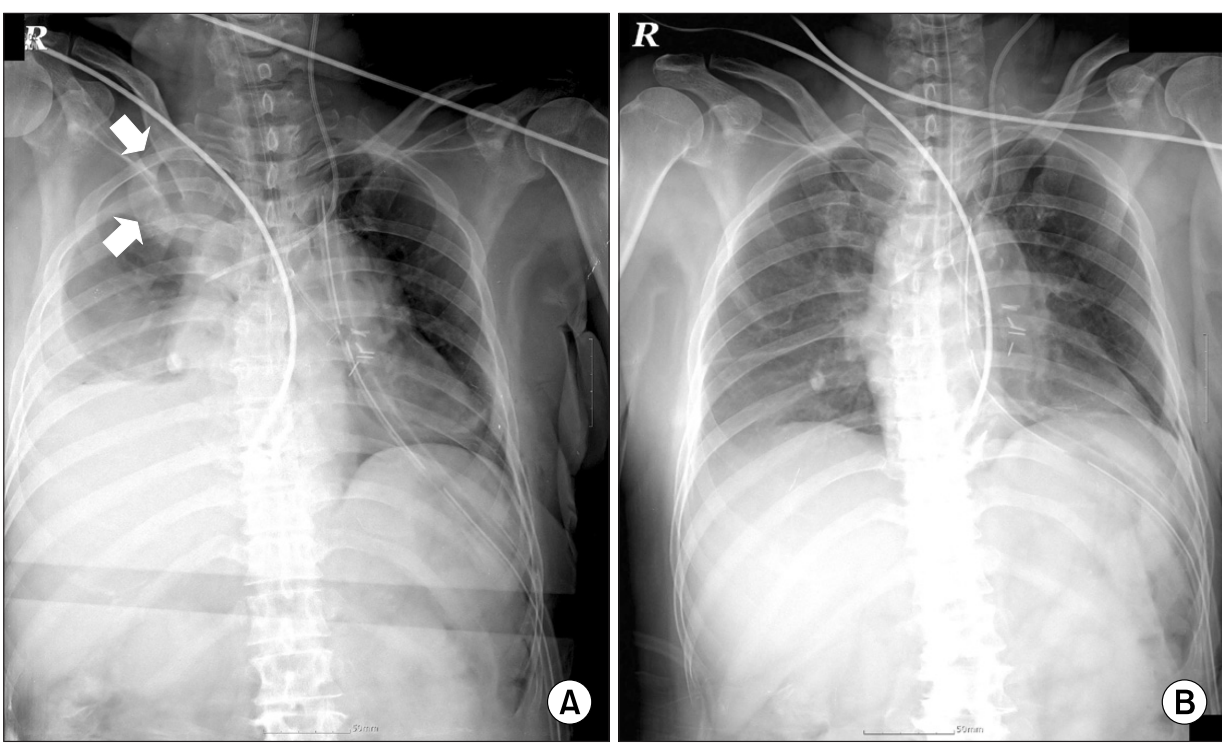

Fig. 1. The chest anteroposterior radiograph taken at the operating room after a left lower lobectomy. (A) Localized right apical lesion. (B) Decreased apical haziness after 10 minutes. 


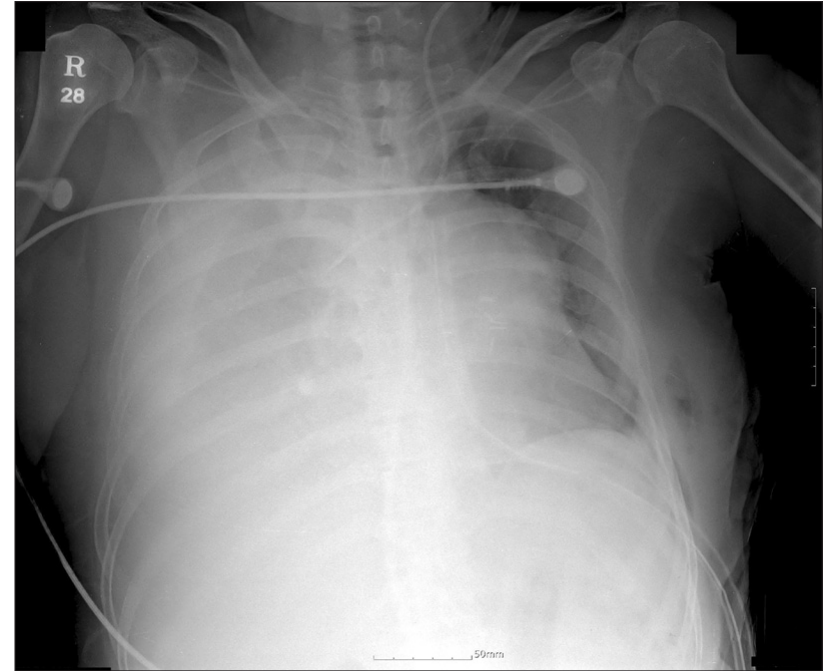

Fig. 2. The chest anteroposterior radiograph taken one day after left lower lobectomy shows right-sided hydrothorax and mediastinal shifting.

in pleural fluid leaking into the chest tube, the patient was moved to the general ward. In the ICU, the CVP was stable at $7-12 \mathrm{mmHg}$ and no changes in the wave forms were observed. Eight days after surgery, the patient had no other complications and was discharged from hospital.

\section{Discussion}

With central venous catheterization, the incidence of mechanical damage, such as pneumothorax and hematothorax, ranges from $3.4 \%$ to $19 \%$ [3], of which a hydrothorax is quite rare at $0.5 \%$ of cases [4]. However, when it does occur, it can cause hypoxemia, as well as cardiovascular collapse and respiratory failure. After inserting the $\mathrm{CVC}$, a hydrothorax and pneumothorax can be caused initially during the process of inserting the CVC, where the blood vessel is perforated. Secondly, while securing the catheter, damage to the blood vessel wall can occur with movement of the catheter [5]. The damage to the blood vessel wall when securing the catheter can be caused by the movement of the patient's head or neck, respiratory movement, or by erosive damage to the blood vessel wall $[2,4,6]$. The right internal jugular vein is connected directly to the superior vena cava, so mechanical damage, such as in this case, are more prevalent during catheterization of the left internal jugular vein than in the right [7]. Colón and Frazier [2] reported a delayed hydrothorax in the case of a CVC in the left subclavian vein, when the hard end of the catheter lacerated the area where the vena cava and the other veins are connected and wore away the blood vessel wall. Flatley and Schapira [6] reported a hydropneumothorax found 7 days after catheterization of the left internal jugular vein. They estimated that catheter movement caused mechanical damage to the blood vessel wall in an area where the blood vessel makes a right angle.

In this case, natural regurgitation in the blood flow and normal CVP waves were observed during surgery. Immediately after inserting the CVC, it was possible to locate the catheter in the vein. One likely cause may be that the catheter had been displaced when the patient's head and neck were moved when the patient was turned to a lateral decuitus position, which caused the catheter to move back and forth. In this process, the catheter moved from the left to the right, perpendicular to the direction of the vein. In addition, cold fluid passed the CVC, increasing its rigidity, which added to the complications. Unusually, the chest X-ray taken immediately after surgery showed the formation of a hydrothorax growing mainly in the right upper lobe. This was attributed to the positioning during surgery. However, a proper early diagnosis was delayed due to confusion with an atelectasis, which is often caused by double-lumen tubes. Moreover, the hydrothorax had progressed with the continual fluid through the CVC. It is also possible for drugs to be administered improperly, as in the case of entirely missing the diagnosis of a hydrothorax caused by the CVC [8].

When hydrothorax due to a CVC is suspected, it is necessary to compare the test results of the pleura fluid seeping in the chest tube and the fluid composition that is injected into the CVC. If the hydrothorax is caused by the CVC, the pleura fluid will effuse with various levels of glucose [3]. In this case, fluid effusion was found in the pleura fluid test, but the location of the CVC appeared normal in the chest X-ray. Therefore, an attempt was made to find the root of the cause in the surgery itself. It was possible to check that the hydrothorax was due to the CVC by injecting indocyanine green directly into the catheter [9]. In this case, we confirmed indirectly when a decrease in the amount of drainage from the thorax was observed after removing the CVC.

To prevent complications from CVC catheterization after insertion, it is necessary to check its condition with serial chest $X$-ray. However, as in this case report, when a small amount of fluid is administered through the CVC and an X-ray is taken immediately after the patient moves from the lateral recumbent position to the supine position, only having an X-ray examination delays a proper diagnosis of a hydrothoraxes localized in the apex of the lung.

CVC catheterization in the internal jugular vein is a basic procedure but complications can occur. Therefore, it is important to be well aware of the anatomical structure and be mindful of complications that can arise. The location of the catheter must be checked with serial X-ray, the aspiration of the CVC must be checked regularly, and the CVP waves must be monitored. Moreover, one should be conscious of the possibility of movement of the end of the catheter due to the movement or changes in the patient's position. When complications do arise, being aware that the cause could be the CVC can reduce the delay in making a proper diagnosis. 


\section{References}

1. Taylor RW, Palagiri AV. Central venous catheterization. Crit Care Med 2007; 35: 1390-6.

2. Colón R, Frazier OH. Right hydrothorax after left subclavian and internal jugular vein catheterization: a delayed complication. Tex Heart Inst J 1985; 12: 389-92.

3. Lee EK. An unexpected left hydrothorax after left internal jugular venous catheterization for total parental nutrition and antibiotics. Ann Acad Med Singapore 2006; 35: 742-4.

4. Bach A. Complications of central venous catheterization. Chest 1993; 104: 654-5.

5. McGee DC, Gould MK. Preventing complications of central venous catheterization. N Engl J Med 2003; 348: 1123-33.
6. Flatley ME, Schapira RM. Hydropneumomediastinum and bilateral hydropneumothorax as delayed complications of central venous catheterization. Chest 1993; 103: 1914-6.

7. Bardosi L, Mostafa SM, Wilkes RG, Wenstone R. Contralateral haemothorax: a late complication of subclavian vein cannulation. Br J Anaesth 1988; 60: 461-3.

8. Kwon YS, Lee SC, Lee EM, Chung MH, Won RS. Hydrothorax and inadvertent administration of thiopental sodium following malpositioned internal jugular vein catheter: a case report. Korean J Anesthesiol 1998; 34: 863-6.

9. Duara R, Rajendran S, Nagi GS, Neelakandhan KS. Hydrothorax following right internal jugular vein cannulation: prompt diagnosis with a simple innovative bedside test. J Postgrad Med 2007; 53: 77-8. 